International Mathematical Forum, 2, 2007, no. 68, 3381 - 3384

\title{
The Metrizablity of Product Spaces
}

\author{
K. Haghnejad Azer
}

\author{
Department of Mathematics \\ Mohaghegh Ardabili University \\ P.O. Box 56199-11367, Ardabil, Iran \\ haghnejadmath@yahoo.com
}

\begin{abstract}
Let $\left(X_{t}\right)_{t \in I}$ be a family of topological spaces and $\prod_{t \in I} X_{t}$ be a product space. In this paper, $\prod_{t \in I} X_{t}$ has two topology which are called product and box topology. We want to establish the metrizablity of $\prod_{t \in I} X_{t}$ with respect to both topology and get some conclusions.
\end{abstract}

Mathematics Subject Classification: 54-xx

Keywords: Metrizablity, Product space, Box topology, Product topology

\section{Introduction}

Given a family $\left(X_{t}\right)_{t \in I}$ of topological spaces, the product spaces of this family is the product set $\prod_{t \in I} X_{t}$ with the topology which is the product of the topologies $X_{t}$. We have two topology in product space $\prod_{t \in I} X_{t}$. First topology is called product topology that has as a base of finite intersection of sets $p_{t}^{-1}\left(U_{t}\right)$, where $U_{t}$ is open set in $X_{t}$ ( $p_{t}$ is the projection function from $\prod_{t \in I} X_{t}$ onto $X_{t}$ such that $\left.p_{t}\left(\left(x_{t}\right)_{t \in I}\right)=x_{t}\right)$. Second topology has base $\prod_{t \in I} U_{t}$ where $U_{t}$ is open set in $X_{t}$ and it is called box topology. In this paper, we establish the metrizablity of $\prod_{t \in I} X_{t}$ with respect to both topologies, that is, for countable set $I$, the space $\prod_{t \in I} X_{t}$ with respect to product topology is metrizable, but is not metrizable whenever $I$ is an uncountable set. In other hand, if $I$ be an infinite set we prove that the space $\prod_{t \in I} X_{t}$ with respect to box topology is not metrizable. Now, we introduce the some notations as follow

We show the set $I$ by natural number or $\{1,2,3, \ldots n\}$ whenever $I$ is countable set. If $(X, d)$ be a metric space and $x_{0} \in X$, the open ball at $x_{0}$ with radius $r>0$ is defined to be the set $B(x, r)=\left\{x \in X: d\left(x, x_{0}\right)<r\right\}$. If $(X, \prec)$ is an order set and $a, b \in X$, we define to be the set $(a, b)=\{x \in X: a \prec x \prec b\}$ $,(a,+\infty)=\{x \in X: a \prec x\},(-\infty, b)=\{x \in X: x \prec b\}$. 


\section{The Metrizablity of product spaces}

Definition 1. If a topology $\tau$ for the set $X$ induced by a metric $d$, we say that $\tau$ and $d$ are compatible with respect to each others.

Definition 2. The set $X$ is metrizable, if the topology $\tau$ over $X$ is compatible with some metric $d$.

Theorem 3. Let $\tau_{1}$ and $\tau_{2}$ be topology over $X$ and let $\beta_{1}$ and $\beta_{2}$ be base for $\tau_{1}$ and $\tau_{2}$,respectively. Then, $\tau_{1} \subseteq \tau_{2}$ if and only if for each $B_{1} \in \beta_{1}$ and $x \in B_{1}$ there exists $B_{2} \in \beta_{2}$ such that $x \in B_{2} \subseteq B_{1}$.

For proof, see[1]

Theorem 4. Let $I$ be a countable set and the topological space $X, \tau)$ be metrizable space (for each $t \in I$ ). Then $\prod_{t \in I} X_{t}$ is metrizable with respect to product topology.

Proof. We show that there is a metric as $D$ such that the product topology induced by its. Let $I$ be an infinite set and $\left(X_{t}, d_{t}\right)$ be a metric space and $\tau_{t}, d_{t}$ are compatible with respect to each others. We define $D_{t}(x, y)=$ $\operatorname{Min}\left\{d_{t}(x, y), 1\right\}$ where $x, y \in X_{t}$, and set $D(x, y)=\sup \left\{\frac{D_{t}(x, y)}{t}: t \in N\right\}$ where $x=\left(x_{t}\right)_{t}$ and $y=\left(y_{t}\right)_{t}$. It is clear that $D$ is a distance function on $X$. Now, we prove that the space $\prod_{t \in I} X_{t}$ is induced by $D$. Let $U$ be an open set with respect to $D$ and $x \in X$ where $x=\left(x_{t}\right)_{t}$. Then there exists $\varepsilon>0$ such that $B_{D}(x, \epsilon) \subset U$. For this $\varepsilon>0$ there is natural number $m$ such that $\frac{1}{m}<\varepsilon$. We define $V=\prod_{i=1}^{m} B_{D_{t}}\left(x_{t}, \varepsilon\right) \times R \times R \times \ldots$, which is neighborhood of $x$ with respect to product topology. We show that $V \subseteq B_{D}(x, \epsilon)$. If $y \in V$ where $y=\left(y_{t}\right)_{t}$, then $\frac{D_{t}\left(x_{t}, y_{t}\right)}{t} \leq \frac{1}{m}$ for all $t \geq m$. Consequently, we have

$$
D(x, y) \leq \operatorname{Max}\left\{\frac{D_{1}\left(x_{1}, y_{1}\right)}{1}, \frac{D_{2}\left(x_{2}, y_{2}\right)}{2}, \frac{D_{3}\left(x_{3}, y_{3}\right)}{3}, \ldots, \frac{D_{m}\left(x_{m}, y_{m}\right)}{t}, \frac{1}{m}\right\}
$$

which implies $y \in B_{D}(x, \epsilon)$. Therefore, $V \subseteq B_{D}(x, \epsilon)$. In other hand, let $U=\prod_{t \in I} U_{t}$ be an open set with respect to product topology on $\prod_{t \in I} X_{t}$ such that $U_{t}=X_{t}$ for all $t \neq \alpha_{1}, \alpha_{2}, \alpha_{3}, \ldots, \alpha_{n}$ and let $x \in U$ be an arbitrary and choosen $\varepsilon_{i}>0$ such that $B_{d_{\alpha_{i}}}\left(x_{\alpha_{i}}, \varepsilon_{i}\right) \subseteq U_{\alpha_{i}}$. Then, we put $\varepsilon=\operatorname{Min}\left\{\frac{\varepsilon_{i}}{i}: i=\alpha_{1}, \alpha_{2}, \alpha_{3}, \ldots, \alpha_{n}\right\}$ whivh implies $B_{D}(x, \epsilon) \subseteq U$. So the application of the Theorem 3 shows that $\prod_{t \in I} X_{t}$ is metrizable with respect to product topology. 
Corollary 5. If $(X, d)$ is a metric space, then the space $X^{N}$ is metrizable with respect to product topology.

Example 6. The space $R^{N}$ is metrizable with respect to product topology.

Theorem 7. Let $(X, d)$ be metric space and $E \subseteq X$ and let $a$ be a limit point of $E$ then there exists a sequence $\left(a_{n}\right)_{n}$ of $E$ converges to $a$.

For proof see $[1,2.10]$

Definition 8. Suppose that $(X, \prec)$ is totally ordered and $x \prec y$. If there exist $a, b, c \in X$ such that $a \prec x \prec b \prec y \prec c$ then $(X, \prec)$ is called $H$-order.

Theorem 9. Let $I$ be an uncountable set, and the topological space $\left(X_{t}, \tau_{t}\right)$ be metrizable. If $\left(X_{t}, \tau_{t}\right)$ is a $H$-order with respect to $\prec_{t}$, then $\prod_{t \in I} X_{t}$ with respect to product topology is not metrizable.

Proof. We prove that the theorem 7 is not hold with respect to product topology for $\prod_{t \in I} X_{t}$. Let $a=\left(a_{t}\right)_{t}$ be a member fix of $\prod_{t \in I} X_{t}$. We define the set as

$$
A=\left\{\left(x_{t}\right)_{t \in I}: \text { except for finite index of }, x_{t} \neq a_{t}\right\} \text {. }
$$

We show that $a$ is belong to $A^{\prime}$ (the limit points of $A$ ). Get $\prod_{t \in I} U_{t}$ be a neighborhood of $a$ such that $U_{t}=X_{t}$ for all $t \neq t_{1}, t_{2}, t_{3}, \ldots, t_{m}$. We choose the element $\left(y_{t}\right)_{t}$ of $A \cap \prod_{t \in I} U_{t}$ such that $y_{t}=a_{t}$ for $t=t_{1}, t_{2}, t_{3}, \ldots, t_{m}$ and otherwise $y_{t} \in X_{t}$. Then, $\left(y_{t}\right)_{t} \in A \cap \prod_{t \in I} U_{t}$ implies $a \in A^{\prime}$.

We claim that there is not any sequence in $A$ such that converges to $a$. Assume that our claim is false then there is a sequence $\left(b_{n}\right)_{n=1}^{\infty}$ of $A$ such that converges to $a$ whenever $b_{n}=\left(b_{n, t)}\right)_{t}$. We define $I_{n}=\left\{t \in I: b_{(n . t)}=a_{t}\right\}$, and set $J=\bigcup_{n=1}^{\infty} I_{n}$. Since the set $I_{n}$ is finite then $J$ is countable set. Hence, there is $\alpha \in I-J$ such that $b_{(n . \alpha)} \prec a_{\alpha}$ for all $n \in N$. Without lose generally, we set $b_{(n . \alpha)}=a_{\alpha}$ (for some $\left.n \in N\right)$. We choose $U=p^{-1}\left(b_{(n . \alpha)},+\infty\right)$ which is a neighborhood of $a$. Then $U$ is not including any $b_{n}$. That is contradiction so the application of Theorem 7 shows that $\prod_{t \in I} X_{t}$ is not metrizable.

Corollary 10. Let $(X, d)$ be a metric space and $I$ be an uncountable set, then the space $X^{I}$ with respect to product topology is not metrizable.

Example 11. Let $I$ be an uncountable set then the space $R^{I}$ with respect to product topology is not metrizable .

Theorem 12. Let $I$ be an infinite countable set and let the topological space $\left(X_{t}, \tau_{t}\right)$ be metrizable. If $X_{t}$ is $H-o r d e r$ with respect to $\prec_{t}$ then $\prod_{t \in I} X_{t}$ 
is not metrizable with respect to box topology.

Proof. Let $a=\left(a_{t}\right)_{t}$ be an arbitrary element of $\prod_{t \in I} X_{t}$. we define the set as $A=\left\{\left(x_{t}\right)_{t}: a_{t} \prec x_{t}\right\}$. First, we show that $a$ is limit point of $A$. We set $U=\prod_{t=1}^{\infty}\left(b_{t}, c_{t}\right)$ which is neighborhood of $a$ whenever $b_{t} \prec_{t} a_{t} \prec_{t} c_{t}$. Since $X_{t}$ is $H$-order then there exists $d_{t} \in X_{t}$ such that $b_{t} \prec_{t} d_{t} \prec_{t} a_{t}$. We conclude that $\left(d_{t}\right) \in U \cap A$. This conclusion shows $a \in A^{\prime}$. Now, we claim that there is not any sequence in $A$ which converges to $a$. For proven of this claim, we get the sequence as $\left(b_{n}\right)_{n}$ which converges to $a$ whenever $\left.b_{n}\right)=\left(a_{(t, n)}\right)_{t}$. Since $X_{t}$ is $H-$ order, there is $c_{t} \in X_{t}$ such that $a_{t} \prec_{t} c_{t} \prec_{t} b_{(t, t)}$. Let choose the neighborhood of $a$ as $V=\prod_{t=1}^{\infty}\left(-\infty, c_{t}\right)$. Then, this neighborhood is not including any $a_{n}$ that is contradiction. Then, the application of theorem7 shows that $\prod_{t \in I} X_{t}$ is not metrizable.

Corollary 13. If $(X, d)$ be a metric space then the space $X^{N}$ with respect to box topology is no metrizable.

Example 14. The space $R^{N}$ with respect to box topology is not metrizable.

Corollary 15. Let $I$ be a countable set and $\left(X_{t}, \tau_{t}\right)$ be a topological space whenever $t \in I$. Let $\left(a_{n}\right)_{n}$ be a sequence in $\prod_{t \in I} X_{t}$ such that $p_{t}\left(\left(a_{n}\right)_{n}\right)$ converges to some point of $X_{t}$ for all $t \in I$. Then, the sequence $\left(a_{n}\right)_{n}$ converges with respect to product topology and divergence with respect to box topology.

problem16. For proven theorems 9 and 12, we used by definition of $H-$ order. We design this question that what will be our answer for these theorems, when we remove the $H$-order condition?

\section{References}

[1] Munkres J. R, Topology a First Courses, Prentice-Hall Inc., Englewood Cliffs Newjersey (1975).

[2] Rudin W., Principle of Mathematical Analysis .Ed.3, Pub. McGrawHill(1976).

[3] Rudin W., Functional Analysis, Mc. Grow-Hill, I.C New York (1973).

Received: August 14, 2007 